\title{
UNA APROXIMACIÓN SOCIOLINGÜÍSTICA AL EMPLEO DEL PRESENTE HISTÓRICO EN NARRACIONES DE EXPERIENCIA PERSONAL EN HABLANTES DE SANTIAGO DE CHILE*
}

\author{
A SOCIOLINGUISTIC APPROACH TO THE USE OF THE \\ HISTORICAL PRESENT IN PERSONAL EXPERIENCE NARRATIVES \\ IN SPEAKERS FROM SANTIAGO, CHILE
}

\author{
SILVANA GUERRERO GONZÁLEZ \\ Universidad de Chile. Santiago, Chile \\ Pontificia Universidad Católica de Chile. Santiago, Chile \\ siguerrero@u.uchile.cl \\ smguerrero@uc.cl
}

\section{RESUMEN}

En el marco del enfoque variacionista laboviano, en esta investigación se analiza el empleo del presente histórico en una muestra de 192 narraciones de experiencia personal del español hablado en Santiago de Chile. Específicamente, se propone establecer la frecuencia de empleo de este elemento variable y comprobar si existe variación entre los factores sociodemográficos -sexo, edad y grupo socioeconómico de los hablantes- y el empleo del mencionado recurso. Asimismo, se pretende describir su ubicación en la estructura narrativa (Labov \& Waletzky, 1967; Labov, 1983 [1972]) y la función que cumple en los relatos. El análisis de los datos demuestra, en primer lugar, que existe variación sociolingüística en el empleo de este recurso en las narraciones de experiencia personal, en concreto, en las mujeres de 20 a 34 años y las de 55 años y más de los cuatro grupos socioeconómicos en estudio, las que presentan significatividad estadística; en segunda instancia, que es posible considerar el presente histórico como un fenómeno variable con una función específica, a saber, la de ser un recurso de evaluación (Labov, 1983 [1972]; Silva-Corvalán, 1983, 2001) y, finalmente, que este fenómeno se ubica en la complicación de la narración (SilvaCorvalán, 1983, 2001).

Palabras clave: Variación discursiva, narración de experiencia personal, presente histórico, recursos evaluativos.

\footnotetext{
* La investigación se desarrolla en el marco de los estudios que está realizando el grupo de Estudio Sociolingüístico del Español de Santiago de Chile (ESECH) del Departamento de Lingüística de la Universidad de Chile y del grupo PRESEEA-SA (Proyecto para el Estudio Sociolingüístico del Español de España y América, equipo de Santiago de Chile).
} 


\begin{abstract}
Following the Labovian variationist approach, this research study analyzes the use of the historical present in a sample of 192 oral personal experience narratives in the Spanish spoken in Santiago, Chile. We aim at establishing the frequency of use of this variable element and testing whether there exists variation among the sociodemographic factors - sex, age, and socioeconomic group- of the speakers and the use of the said resource. We also describe its place in the narrative structure (Labov \& Waletzky, 1967; Labov, 1983 [1972]) and the function it performs in the narratives (Silva-Corvalán, 1983, 2001). Our data analysis shows that, firstly, there is sociolinguistic variation in the use of the historical present in personal experience narratives, specifically, among women of 20 to 34 years and over 55 in the four socioeconomic groups studied, presenting statistical significance. Secondly, our analysis indicates that it is possible to consider the historical present as a variable phenomenon with a specific function, namely, that of being an evaluative resource (Labov, 1972; Silva-Corvalán, 1983, 2001). Finally, we could establish that this phenomenon is located in the narrative complication (Silva-Corvalán, 1983, 2001).

Keywords: Discourse variation, personal experience narratives, historical present, evaluative resources.
\end{abstract}

Recibido: 28.08.2013. Aceptado: 30.06.2014.

\title{
1. INTRODUCCIÓN
}

\subsection{Naturaleza, alcance, objetivos e hipótesis del estudio}

- 1 creciente interés por el estudio de la variación sintáctica y discursiva en esCañol ha dado origen a numerosas publicaciones que, desde diferentes puntos de vista, se han aproximado al estudio de variables lingüísticas a nivel de la sintaxis y el discurso. Algunos aspectos de la variación sintáctica y discursiva en el habla de Santiago de Chile han sido estudiados por Prieto (1995-1996), Poblete (1998), Silva-Corvalán (2001), Prieto y San Martín (2002-2003), San Martín (2004-2005, 2011, 2013), Guerrero (2011a, 2011b), Rojas, Rubio, San Martín y Guerrero (2012) y San Martín y Guerrero (2013). En este contexto, en esta investigación se propone analizar el empleo del presente histórico en una muestra de narraciones de experiencia personal en el español hablado en Santiago de Chile. A su vez, los objetivos específicos de este trabajo son, por una parte, establecer la frecuencia de empleo del presente histórico y, por otra, comprobar si existe variación entre los factores sociodemográficos de los hablantes y el empleo del elemento variable analizado. Asimismo, en términos cualitativos, se propone describir la ubicación dentro de la estructura narrativa y la función que cumple 
el elemento variable en estudio en las narraciones. La hipótesis de trabajo es que las características sociodemográficas de los hablantes de español en Santiago de Chile están correlacionadas con el empleo del presente histórico en las narraciones de experiencia personal. Para tales propósitos se analizan 192 narraciones de sujetos estratificados socioculturalmente, conformando, de esta forma, una muestra significativa de discurso natural grabado en el estilo que Labov denomina como vernacular (Labov, 1983 [1972], 1978).

El marco de esta investigación es la sociolingüística, en particular, desde el enfoque variacionista. En dicho enfoque, los procedimientos de análisis estadístico permiten establecer correlaciones entre el comportamiento lingüístico y los factores sociodemográficos de los hablantes. En este sentido, el trabajo se hace parte en el debate que desde la década de los setenta se ha generado en el campo de la sociolingüística en torno a si es posible extender el concepto de variable a otros planos de la lengua más allá del nivel fonético, específicamente, aludiendo a un elemento estructural de las narraciones, que por manifestarse de manera variable puede cuantificarse. Asimismo, la investigación aquí proyectada se perfila como una contribución a la descripción sociolingüística del español hablado en Santiago de Chile.

\section{MARCO TEÓRICO}

\subsection{El concepto de variable sociolingüistica y su aplicación al presente histórico}

Dentro de la sociolingüística variacionista, una variable sociolingüística es un fenómeno de variación lingüística en que los valores o variantes de la variable están correlacionados con alguno de los factores sociodemográficos de los hablantes o de la situación de habla, así, la variación sociolingüística ha sido definida como "la alternancia de dos o más expresiones de un mismo elemento, cuando ésta no supone ningún tipo de alteración o cambio de naturaleza semántica y cuando se ve condicionada por factores lingüísticos y sociales" (Moreno Fernández, 2009: 39).

Los análisis basados en el método variacionista en el plano fonético-fonológico de la lengua se llevaron a cabo con gran éxito, muestra de ello son, por ejemplo, los trabajos de Labov sobre la estratificación de los diptongos /ay/ y /aw/ en el inglés de Martha’s Vineyard, o la estratificación social de /r/ en el inglés de Nueva York. Sin embargo, la extensión del concepto de variable sociolingüística al estudio de fenómenos de variación sintáctica se tradujo en un conjunto de críticas, especialmente, a partir del trabajo de Lavandera (1984 [1978]). Las críticas de la investigadora se basaban especialmente en que en el estudio de dichas variables no estaba garantizado el principio de equivalencia semántica de Labov, indispensable para hablar de una variable sociolingüística. Lo mismo, y con mayor grado de comple- 
jidad aún, se daría para la variación a nivel de discurso, que es donde se inserta el fenómeno que analizamos. Así, la variación lingüística "definida como el uso alterno de formas diferentes de decir lo mismo, se puede encontrar prácticamente en todos los niveles de la lengua, desde el más concreto (fonético-fonológico) al más amplio (discurso, por ejemplo), pasando por la gramática y el léxico" (Moreno Fernández, 2009: 32). Por consiguiente, la ampliación de los estudios variacionistas al plano sintáctico y discursivo es, en la actualidad, considerablemente aceptada, por lo que se hace alusión a la existencia de una sociolingüística post-laboviana (Moreno Fernández, 2012).

En relación con el fenómeno que atañe a esta investigación, se destaca que no es el objetivo estudiar las formas de referirse al pasado como una variable, y el pretérito y el presente histórico como sus variantes, sino que recogiendo los antecedentes de los trabajos pioneros de Labov \& Waletzky (1967) y de Labov (1972) se sugiere la existencia de diferencias de disponibilidad de recursos lingüísticos, determinadas por las variables sociales de los informantes, para la construcción de narraciones, en específico, de experiencia personal. En consecuencia, aquí no se hablará de equivalencia semántica, sino de un elemento estructural de las narraciones, que se manifestaría de manera variable y que, por ende, puede cuantificarse. Así, se aludirá a la variación en términos genéricos y no como venía haciéndose fundamentalmente en los setenta, en términos de variable y variantes (cf. Guerrero, 2013).

\subsection{La variación según variables sociodemográficas: sexo, edad y grupo socioeconómico}

El desarrollo de la sociolingüística se basa en que gran parte de los hechos de variación lingüística varían en forma sistemática, si se los correlaciona con variables sociodemográficas como el grupo socioeconómico, la edad y el sexo de los informantes. Esto implica que el hecho de que las variables lingüísticas estén en covariación sistemática con las variables permite a los individuos instalarse en un determinado espacio del espectro social. En relación con esto, Coates (2009: 89) pone de relieve que "aunque desde el punto de vista lingüístico las variables lingüísticas sean formas equivalentes para referirse a algo en particular, son diferentes desde una perspectiva social".

Por su parte, según Moreno Fernández (1998), la investigación sociolingüística ha demostrado que las variables sociales que influyen sobre la variación lingüística lo hacen de una forma específica en cada comunidad de habla y respecto de fenómenos lingüísticos concretos. En este orden, Blas Arroyo (2005) también señala que no es posible reconocer de antemano qué tipo de variables sociales van a actuar sobre la variación lingüística, primero, porque la variabilidad no tiene por qué manifestarse de la misma forma en comunidades de habla diferentes y, segundo, 
porque estos mismos factores no se configuran de la misma forma en sociedades distintas. Como ya se señaló, los factores sexo, edad y grupo socioeconómico serán las variables independientes empleadas en esta investigación sobre el carácter que asume la variación lingüística correspondiente al empleo del presente histórico en una muestra del español hablado en Santiago de Chile.

\subsection{El presente histórico en el discurso narrativo}

La idea que subyace a la literatura referida al presente histórico es que se trata de un uso particular de las formas de presente para hacer referencia a situaciones pasadas (miro por miré). En concreto, Alarcos Llorach (1994: 156) señala que "se llama presente histórico al empleo, tanto en la narración escrita como en el vivo relato coloquial, de las formas de presente para aludir a hechos cronológicamente ocurridos en el pasado". Asimismo, los estudios sobre presente histórico concuerdan en que este uso particular del presente indicativo tiene efectos estilísticos en el discurso, dotándolo de mayor fuerza, viveza y dramatismo, y denominándolo, en consecuencia, "presente teatralizado" (cf. Bello, 1841; Gili Gaya, 1961; Chafe, 1982; Calsamiglia y Tusón, 1999; y Avilés y Osorio, 2010, entre otros). La Nueva gramática de la lengua española (2009) incorpora la diferencia postulada entre los usos del presente histórico en discursos escritos y discursos orales, distinguiendo entre el "presente histórico", característico de las biografías y de las descripciones históricas, cuyo rasgo principal es la posibilidad de trasladar el punto del habla a un momento del pasado, y el "presente narrativo", propio de las narraciones y compatible con los eventos referidos en pasado. Es este último el que nos interesa particularmente en tanto fenómeno variable en la secuencia narrativa.

Como ya se señaló, situamos nuestro estudio del presente histórico en la narración de experiencia personal. La bibliografía sobre narraciones es extensa; no obstante, las investigaciones pioneras de Labov \& Waletzky (1967) y de Labov (1972), han demostrado ser el modelo de más alto rendimiento empírico en la investigación sobre el problema, como ha quedado de manifiesto en varios estudios sobre narrativas (Reyes, 1996, 2002, 2003; Soler, 2004; Jiménez, 2006; y Shiro, 2007, por solo citar algunos ejemplos). Labov (1972: 360) define la narración como "one method of recapitulating past experience by matching a verbal sequence of clauses to the sequence of events which (it is inferred) actually occurred [un método de recapitulación de la experiencia pasada adecuando una secuencia verbal de proposiciones a la secuencia de sucesos que (se supone) ocurrieron realmente (la traducción es nuestra)]". La narración es considerada como un texto que expresa una serie de eventos que ocurren en el tiempo, pero donde, a su vez, se percibe de manera subyacente una relación causal o temática. Labov \& Waletzky (1967) y Labov (1972), distinguen dos tipos de relatos: los relatos mínimos, compuestos 
de -al menos- dos cláusulas narrativas en pasado y los relatos completos. Dichos autores plantean que una narración de experiencia personal plenamente formada exhibe seis rasgos estructurales bien definidos, a saber: 1) resumen, 2) orientación, 3) acción de complicación de la narración, 4) evaluación, 5) resultado o resolución y 6) coda o moraleja. Para los fines de esta investigación, la importancia de los aportes del trabajo de Labov \& Waletzky (1967) y del de Labov (1972) radica en que, por un lado, el presente histórico ha sido considerado un mecanismo de evaluación (cf. 2.3.1) y, por otro, en que una sección de este análisis considerará la frecuencia de aparición del presente histórico en las partes estructurales de la narración.

\subsubsection{La función del presente histórico en las narraciones}

El presente histórico, al alternar con formas verbales en pretérito, conlleva mayores efectos de viveza y dramatismo, cumpliendo una función evaluativa dentro del relato (Silva-Corvalán, 1983; Avilés y Osorio, 2010). En relación con esto, es necesario recordar que Labov (1972) señala que la evaluación no sólo se enmarca en una sección de la narración, sino que es transversal a ésta. Tal como enfatizan Avilés y Osorio (2010: 369, citando a Labov, 1972): "Aun cuando se entiende que la sección de evaluación suspende la acción en el momento de mayor clímax, sería un error limitarla sólo a un par de cláusulas, ya que los dispositivos evaluativos están distribuidos a través de toda la narración”.

A aquellos recursos evaluativos que cumplen la función de suspender la acción, Labov (1972) los denomina dispositivos de "evaluación externa” y los recursos que permiten preservar la continuidad dramática reciben el nombre de mecanismos de "evaluación interna". Al respecto, Silva-Corvalán (1983) plantea que el presente histórico corresponde a un mecanismo de evaluación interna, ya que actúa dentro de la cadena narrativa y se vincula con eventos que transforman la narración en reportable, por lo tanto, responde a una función evaluativa. Asimismo, esta autora comprueba empíricamente que el empleo del presente histórico es más frecuente en la sección de complicación de la narración. En este orden, Chafe (1982) señala que el presente histórico es un rasgo propio del involucramiento y asigna importantes marcas de vivacidad, y Calsamiglia y Tusón (1999) también lo ponen de relieve cuando destacan sus efectos de viveza dentro de la narración.

\subsubsection{Estudios sobre presente histórico en narraciones de experiencia personal en español}

Aunque se trata de un estudio de verbos en general, por su valor de ser pionero en Chile, no se puede dejar de mencionar el trabajo de Valencia y Hermansen (1985), quienes señalan: "Es sabido que el verbo es la categoría de palabra más compleja en 
nuestro sistema lingüístico, tanto por su riqueza significativa como por las diversas señalizaciones que porta" (vii).

Hasta mediados de la década de los 80 , los estudios sobre presente histórico en español se habían sustentado sobre la base de discursos escritos. Más recientemente, trabajos como el de Silva-Corvalán $(1983,2001)$ han señalado la importancia de extender estos estudios al ámbito de los discursos orales, a fin de diferenciar los usos que tiene este recurso en ambas dimensiones, la oral y la escrita. Esta autora enfatiza en que los efectos atribuidos al presente histórico sólo son observables en contextos conversacionales $y$, por consiguiente, tendría una función netamente evaluativa.

En el caso del empleo del presente histórico en el habla de Santiago de Chile se contó con la investigación empírica de Avilés y Osorio (2010), cuyo objetivo consistía en dar cuenta del empleo de esta forma verbal en narraciones de experiencia personal desde tres perspectivas, a saber: 1) sus propiedades témporo-aspectuales, 2) su vínculo con las secciones narrativas propuestas por Labov \& Waletzky (1967) y 3) su contenido significativo desde la Teoría de la relevancia. Esta investigación no considera, sin embargo, la variación sociolingüística en el empleo del presente histórico, foco central de nuestro trabajo.

\section{METODOLOGÍA}

\subsection{Corpus}

El corpus que sirve de base para este estudio corresponde a 192 narraciones de experiencia personal extraídas de entrevistas sociolingüísticas pertenecientes al corpus del grupo de Estudio Sociolingüístico del Español de Chile (ESECH) ${ }^{1}$ realizadas por los estudiantes en la cátedra de Sociolingüística, a cargo del profesor Abelardo San Martín, de las carreras de Licenciatura en Lengua y Literatura Hispánicas y Lengua y Literatura Inglesas de la Universidad de Chile. Con base en el marco de la metodología variacionista propuesta por Labov, las entrevistas fueron realizadas a hombres y mujeres con características sociodemográficas congruentes. En la situación de entrevista, los entrevistadores debían tratar de superar la paradoja del observador ${ }^{2}$ consiguiendo, de esta forma, una muestra significativa

${ }^{1}$ El ESECH, grupo del cual formamos parte, está a cargo del profesor del Departamento de Lingüística de la Universidad de Chile, Abelardo San Martín, a quien agradecemos la facilitación del corpus de esta investigación.

2 Según Labov (1972), el objetivo de la investigación lingüística de una comunidad de habla consiste en estudiar la forma en cómo habla la gente cuando no está siendo sistemáticamente observada; sin embargo, sólo podemos obtener tales datos mediante la observación sistemática. A esto es a lo que se le ha denominado paradoja del observador. Este dilema se soluciona encontrando la manera de completar la entrevista formal con otros datos, o cambiar la estructura de la situación 
de discurso natural grabado (estilo vernáculo ${ }^{3}$ ) de hablantes representativos de la comunidad de habla en estudio.

El cuestionario utilizado para la realización de las mencionadas entrevistas consta de una sección cuya función es elicitar las narraciones de experiencia personal que conforman el corpus de nuestra investigación. En relación con esto, es necesario precisar que se seleccionó la narración mejor desarrollada por cada informante, que es, básicamente, aquella que contiene la mayor cantidad de cláusulas narrativas en términos labovianos. De esta forma, se puede medir el mejor desempeño de cada uno de ellos y, posteriormente, compararlos. Este criterio de selección de la narración mejor desarrollada nos permitió controlar algunas variables intervinientes, como el cansancio y el desinterés de los sujetos considerando la duración de la entrevista, entre otras que pudieran surgir en el marco de la misma.

\subsubsection{Características del corpus utilizado}

Como se señaló anteriormente, la muestra empleada forma parte del corpus del ESECH, cuyo objetivo principal es el estudio de variables sociolingüísticas en el español hablado en Santiago de Chile, y está conformado por 192 entrevistas, realizadas a sujetos santiaguinos ${ }^{4}$ entre los años 2005 y 2010. La estratificación de los sujetos se realizó a base de los criterios metodológicos del ESECH, que considera tres variables: 1) nivel educacional, 2) profesión u ocupación y 3) comuna de residencia.

En este contexto, la entrevista semidirigida es el medio más adecuado para obtener muestras de lengua hablada en la cantidad y con la calidad necesarias para su análisis cuantitativo y, asimismo, es el medio más adecuado para registrar sistemáticamente el habla vernacular, en la que se presta una atención mínima al discurso propio (Moreno Fernández, 2012: 180). Esto implica, por supuesto, no

de la entrevista de una u otra manera. En este sentido, una forma de superar esta paradoja consiste en romper las constricciones de la situación de entrevista mediante diversos procedimientos que distraen la atención del sujeto en su propio discurso y pueden hacer brotar su habla más vernácula.

${ }^{3}$ Citando a Labov, Moreno Fernández (2012: 181-182) señalan: "El vernáculo, en el que se presta al discurso propio una atención mínima, aporta los datos más sistemáticos para el análisis lingüístico. El vernáculo se define como la forma de hablar adquirida durante la preadolescencia. Su carácter altamente regular se ha observado empíricamente. [...] Todo hablante dispone de una forma vernácula al menos en una lengua, que puede ser un dialecto de prestigio [...] o una variedad no estándar. En algunos casos pueden obtenerse datos sistemáticos de estilos de habla más formales, pero no estaremos seguros de ello hasta que no se hayan contrastado con el vernáculo".

${ }^{4}$ Por lo que refiere a los criterios de asignación de hablante nativo de Santiago de Chile, de acuerdo con Prieto (1995-1996: 399), se aplicaron las siguientes restricciones en la selección de los sujetos: 1) haber nacido y residido en forma ininterrumpida en Santiago; 2) haber residido en forma ininterrumpida en Santiago desde los cinco años de edad y 3) haber nacido en Santiago y haber residido en Santiago la mayor parte de sus vidas, salvo por periodos que sumados no superen los cuatro años en el tramo de 55 años y más y los tres años en el tramo de 35 a 54 años. 
olvidar el efecto de la paradoja del observador, por lo que: 1) se procuró generar un clima de confianza con el objetivo de obtener el estilo de habla más espontáneo del sujeto, 2) idealmente, los hablantes debían olvidar que la entrevista estaba siendo grabada, es decir, su comportamiento debía ser lo más natural posible y 3) para conseguir el estilo vernacular esperado, se procuró que la entrevista se realizara en un lugar tranquilo, idealmente en la casa del informante o en un lugar que no le fuera extraño, a fin de garantizar su comodidad.

Cabe señalar, por otro lado, que el cuestionario sólo constituyó una guía para el entrevistador que le permitiera llevar a cabo la conversación de acuerdo con ciertas secciones. En cuanto a su estructura, el cuestionario comprendía varias secciones, de las cuales, es la segunda, narración de experiencias personales, la que empleamos para la conformación del corpus que sirve de base para nuestro estudio.

Por último, para la estratificación de los sujetos que conforman la muestra del estudio, se empleó el sistema de adscripción de estatus social empleada en ESECH que parte de una asignación de puntaje según las siguientes tres variables: 1) nivel educacional, 2) profesión u ocupación y 3) comuna de residencia. A cada una de estas variables se le otorgó una ponderación distinta, a saber: 3 puntos para el nivel educacional, 2 para la variable profesión u ocupación y 1 punto para la variable comuna de residencia. La escala incluye cuatro estratos socioeconómicos, a saber: bajo, medio bajo, medio y medio alto, para cuya diferencia se cauteló la consistencia entre las variables antes mencionadas de manera que respondieran a lo que Prieto (1995-1996), siguiendo a Lenski (1954), denominara congruencia de status.

\subsection{Población y muestra}

En esta investigación se consideró la población constituida por hombres y mujeres de la Región Metropolitana de más de 20 años de edad. Tomando en consideración que tanto la segmentación de la comunidad de habla en grupos socioeconómicos como la división entre hombres y mujeres quedó ya explicada, en lo referido a los tres grupos etarios que hemos considerado apelamos a tres momentos vitales (Blas Arroyo, 2005), es decir, en el primer grupo (20 a 34 años) se encuentran los sujetos que ingresan al mundo laboral o que llevan poco tiempo desempeñando una profesión o actividad específicas, pues a partir de ese momento hay un desarrollo de los patrones "adultos" de variación. Por su parte, en el segundo grupo (35 a 54 años) están incluidos los sujetos que tienen un desarrollo laboral pleno y, finalmente, en el tercer grupo etario (55 años y más) se hallan los sujetos que están prontos a dejar el mundo laboral o que ya lo han dejado. En este sentido, se trabaja con etapas por las que pasa el hablante, que suelen estar vinculadas con la cultura de cada comunidad lingüística (Serrano, 2011). 
El cuestionario se aplicó a una muestra del tipo "muestra por cuotas con afijación uniforme", en la que se divide a la población en estratos o categorías y se asigna una cuota a cada uno de los distintos estratos (López Morales, 1994: 58). La muestra, así conformada, comprende un total de 192 entrevistas realizadas a igual número de sujetos, distribuidos como se indica en la Tabla I.

Tabla I. Proporción de sujetos según características sociodemográficas de sexo, edad y grupo socioeconómico.

\begin{tabular}{|l|c|c|c|c|c|c|c|}
\hline & \multicolumn{2}{|c|}{$20-34$} & \multicolumn{2}{c|}{$35-54$} & \multicolumn{2}{c|}{55 y más } & \\
\hline & $\mathrm{H}$ & $\mathrm{M}$ & $\mathrm{H}$ & $\mathrm{M}$ & $\mathrm{H}$ & $\mathrm{M}$ & Total \\
\hline Medio alto & 8 & 8 & 8 & 8 & 8 & 8 & $=48$ \\
\hline Medio & 8 & 8 & 8 & 8 & 8 & 8 & $=48$ \\
\hline Medio bajo & 8 & 8 & 8 & 8 & 8 & 8 & $=48$ \\
\hline Bajo & 8 & 8 & 8 & 8 & 8 & 8 & $=48$ \\
\hline & 32 & 32 & 32 & 32 & 32 & 32 & $=192$ \\
\hline
\end{tabular}

\section{PRESENTACIÓN Y ANÁLISIS DE LOS RESULTADOS}

\subsection{Análisis variacionista del presente histórico en el corpus}

El presente histórico se presentó en 351 ocasiones en nuestro corpus, distribuido como se muestra en la Tabla II, que presentamos a continuación.

Tabla II. Distribución del empleo del presente histórico en el corpus.

\begin{tabular}{|c|c|c|c|c|c|c|}
\hline Grupo Socioeconómico & & $20-34$ & $35-54$ & $55 y+$ & Subtotales & Totales \\
\hline \multirow[b]{2}{*}{ MA } & $\mathrm{H}$ & \begin{tabular}{|l|}
0 \\
$(0,0)$
\end{tabular} & $\begin{array}{l}5 \\
(1,4)\end{array}$ & $\begin{array}{l}4 \\
(1,1)\end{array}$ & $9(2,6 \%)$ & \multirow{2}{*}{$\begin{array}{l}45 \\
(12,8 \%)\end{array}$} \\
\hline & M & $\begin{array}{l}22 \\
(6,2)\end{array}$ & \begin{tabular}{|l|}
13 \\
$(3,7)$
\end{tabular} & $\begin{array}{l}1 \\
(0,2)\end{array}$ & $36(10,2 \%)$ & \\
\hline \multirow[b]{2}{*}{ M } & $\mathrm{H}$ & $\begin{array}{l}19 \\
(5,4) \\
\end{array}$ & $\begin{array}{l}7 \\
(1,9) \\
\end{array}$ & $\begin{array}{l}15 \\
(4,2) \\
\end{array}$ & $41(11,7 \%)$ & \multirow{2}{*}{$\begin{array}{l}100 \\
(28,6 \%)\end{array}$} \\
\hline & $\mathrm{M}$ & $\begin{array}{l}19 \\
(5,4) \\
\end{array}$ & $\begin{array}{l}3 \\
(0,8) \\
\end{array}$ & $\begin{array}{l}37 \\
(10,5) \\
\end{array}$ & $59(16,9 \%)$ & \\
\hline & $\mathrm{H}$ & $\begin{array}{l}24 \\
(6,8) \\
\end{array}$ & $\begin{array}{l}14 \\
(4) \\
\end{array}$ & $\begin{array}{l}5 \\
(1,4)\end{array}$ & $43(12,3 \%)$ & \multirow{2}{*}{$\begin{array}{l}110 \\
(31,3 \%)\end{array}$} \\
\hline $\mathrm{MB}$ & $\mathrm{M}$ & $\begin{array}{l}12 \\
(3,4) \\
\end{array}$ & \begin{tabular}{|l}
17 \\
$(4,8)$ \\
\end{tabular} & $\begin{array}{l}38 \\
(10,9)\end{array}$ & $67(19 \%)$ & \\
\hline
\end{tabular}


Una aproximación sociolingüística al empleo del presente histórico en narraciones de experiencia personal en... / S. GUERRERO

Continuación Tabla II.

\begin{tabular}{|c|c|c|c|c|c|c|}
\hline & $\mathrm{H}$ & $\begin{array}{l}21 \\
(6)\end{array}$ & \begin{tabular}{|l|}
44 \\
$(12,5)$
\end{tabular} & $\begin{array}{l}6 \\
(1,7)\end{array}$ & $71(20,2 \%)$ & \multirow{2}{*}{$\begin{array}{l}96 \\
(27,3 \%)\end{array}$} \\
\hline B & M & $\begin{array}{l}19 \\
(5,4)\end{array}$ & $\begin{array}{l}6 \\
(1,7)\end{array}$ & $\begin{array}{l}0 \\
(0,0)\end{array}$ & $25(7,1 \%)$ & \\
\hline & & $\begin{array}{l}136 \\
(38,7 \%)\end{array}$ & $\begin{array}{l}109 \\
(31,1 \%)\end{array}$ & \begin{tabular}{|l|}
106 \\
$(30,2 \%)$
\end{tabular} & $\begin{array}{l}351 \\
(100 \%)\end{array}$ & \begin{tabular}{|l|}
351 \\
$(100 \%)$
\end{tabular} \\
\hline
\end{tabular}

Como puede observarse en la Tabla II, son los hombres de 35 a 54 años del grupo socioeconómico bajo los que presentan la mayor frecuencia de empleo del presente histórico (12,5\%). Le siguen las mujeres del grupo medio bajo de $55 \mathrm{y}$ más años $(10,9 \%)$ y las mujeres del grupo medio del mismo grupo etario (10,5\%). Asimismo, se observa que son los sujetos del grupo etario de 20 a 34 años de los cuatro grupos socioeconómicos los que se mantienen más constantes en el uso del mencionado recurso y que, en total, son los que registran la mayor frecuencia de empleo $(38,7 \%$ versus $31,1 \%$ en el grupo intermedio y $30,2 \%$ en el tercer grupo), excepto por los hombres del grupo medio alto que no utilizan el presente histórico. De hecho, esta característica es muy interesante en la medida en que observamos que tampoco las mujeres del grupo bajo del tercer grupo etario emplean esta forma de pasado, por lo tanto, es en los extremos de la escala socioeconómica donde disminuye su uso, esto es, en los hombres del grupo medio alto $(2,6 \%$ en total) y en las mujeres del grupo bajo (7,1\% en total) lo que incide, a su vez, en el reporte total de ambos grupos socioeconómicos. Otro dato relevantes es que solo en el estrato bajo, los hombres superan a las mujeres de los tres grupos etarios en el uso del presente histórico $(20,2 \%)$ y son, de hecho, los que más lo usan en el corpus.

Para efectos del análisis estadístico, se consideró que dada la distribución no normal de los datos lo más conveniente era emplear estadística no paramétrica. Los datos obtenidos en el análisis de su comportamiento mostraron que el 59,9\% $(115 / 192)$ de los informantes no utiliza el mencionado recurso evaluativo y el $40,1 \%$ restante (77/192) lo emplea entre 1 y 32 ocasiones, concentrándose los porcentajes de frecuencias más elevados en la frecuencia 1 utilizada por el 10,9\% (21/192) de los informantes, y 2 en un 8,3\% (16/192) de los sujetos que componen la muestra. Por su parte, el Histograma generado da cuenta de la dispersión de los datos de la muestra, donde la media aritmética es de 1,83 y la desviación típica de 3,967, por lo tanto, es preciso llevar a cabo un análisis de estadística no paramétrica, en concreto, una prueba de ji cuadrado, que correlacione la ausencia o la presencia de la variable en estudio con los factores sociodemográficos de los sujetos. En este caso, la significatividad estadística, esto es, que la diferencia no sea aleatoria sino que refleje la posibilidad de que se diera por azar, es $\mathrm{p}<0,05$. 


\subsubsection{Incidencia del factor sexo en el empleo del presente histórico}

Como ya se señaló, el presente histórico se registró en 351 ocasiones en nuestro corpus. Esta frecuencia se distribuyó, según el factor sexo, como sigue: 46,7\% (164/351) en el caso de los hombres y 53,3\% (187/351) en las mujeres. No obstante, en el total de la muestra sólo se presenta en 77/192 (40,1\%) informantes, es decir, en menos de la mitad de las narraciones.

Por su parte, la prueba ji cuadrado aplicada a la interacción de la variable sexo con el empleo del presente histórico, da cuenta de que no hay significación estadística, $\mathrm{p}=0,659$. Como se observa, la prueba aplicada demuestra que el resultado de $\mathrm{p}$ es superior a 0,05 , por lo tanto, los efectos de azar en la correlación del factor sexo y el empleo del presente histórico son muy altos. Se presenta el Gráfico 1, que ilustra los datos derivados de la tabla de contingencia correspondiente.

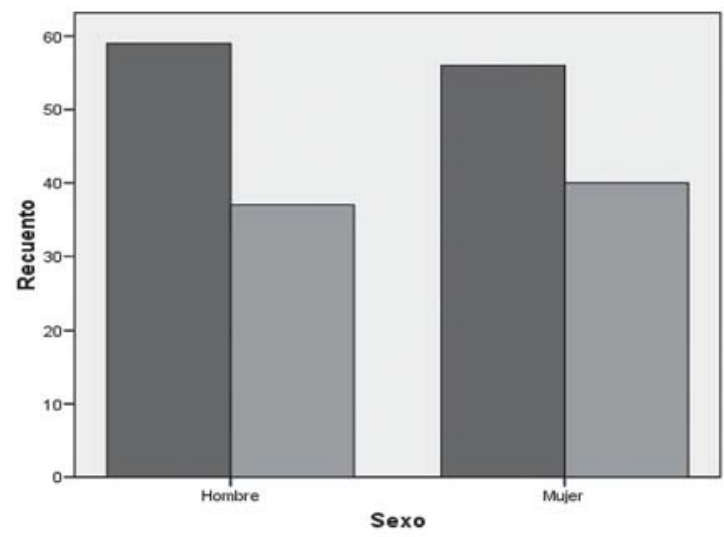

Gráfico 1. Presencia y ausencia de presente histórico en las narraciones de la muestra según el factor sexo.

El Gráfico 1 demuestra que el fenómeno en estudio manifiesta menor presencia que ausencia tanto en hombres como en mujeres. Pese a que la significación no es estadística, se destaca el aumento de presencia -independiente de la frecuencia absoluta descrita con anterioridad- en las mujeres que conforman la muestra, quienes lo emplean en el 52\% (40/77) de las narraciones, mientras que los hombres lo utilizan en el 48\% (37/77) de los casos. Esa diferencia, aunque mínima, le otorga la mayor frecuencia absoluta de empleo a las mujeres, las que, como se muestra en la Tabla II, excepto en el grupo socioeconómico bajo, supe- 
ran a los hombres en el uso del presente histórico, dotando a las narraciones de mayor dinamismo y vivacidad, porque, se debe recordar, esa es parte de la función del componente evaluativo en el relato: ser un mecanismo lingüístico que permite justificar la narración (Labov \& Waletzky, 1967; Labov, 1972). En esta misma línea, Brown y Levinson (1987: 106) proponen intensificar el interés del otro como una de las estrategias para contribuir a la cortesía positiva. Una de las formas de lograr este objetivo es precisamente haciendo una buena historia. Así, los autores reconocen que una forma de construir una buena historia es empleando el presente histórico.

\subsubsection{Incidencia del factor edad en el empleo del presente histórico}

En lo que respecta a la prueba ji cuadrado, tal como ocurrió con el factor sexo, en los tres grupos etarios la interacción no es significativa $(\mathrm{p}=0,511)$. De las 351 ocasiones en que se registra el fenómeno, $136(38,7 \%)$ son utilizadas por los hablantes de 20 a 34 años, $109(31,1 \%)$ por los informantes del grupo etario intermedio y $106(30,2 \%)$ por los hablantes de 55 años y más. Los antecedentes derivados de la tabla de contingencia de donde se obtiene el valor de p pueden verse ilustrados en el Gráfico 2, que se comenta posteriormente.

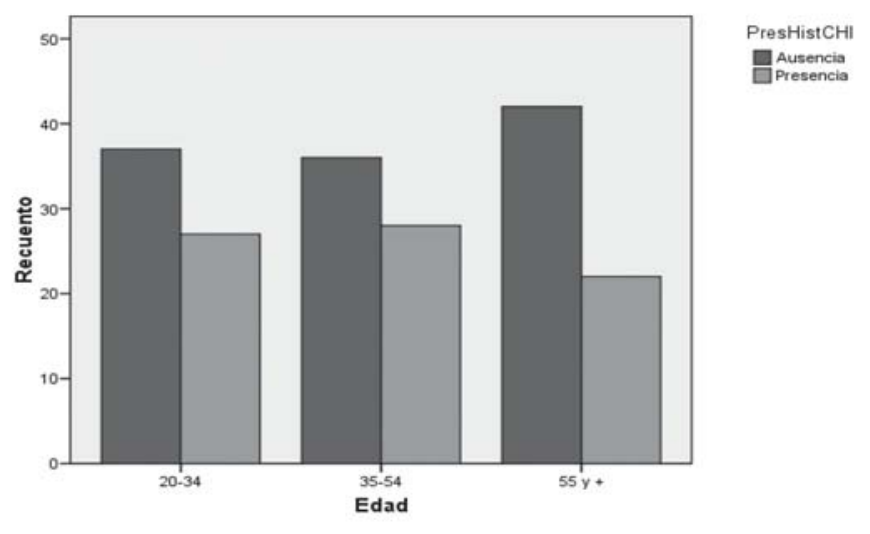

Gráfico 2. Presencia y ausencia de presente histórico en las narraciones de la muestra según el factor edad.

El Gráfico 2 contiene la información sobre la presencia y la ausencia del empleo del presente histórico en el corpus en correlación con el factor edad. Como es evidente, la presencia del fenómeno es mucho menor que la ausencia en los tres 
grupos en estudio. En el caso de los informantes que lo emplean, un 35\% (27/77) corresponde a los del primer grupo etario, un 36,4\% (28/77) a los del grupo de edad intermedia y un $28,6 \%(22 / 77)$ a los del tercer grupo etario. Estas diferencias, como ya se señaló, no constituyen significación estadística; sin embargo, se debe destacar la disminución del empleo de presente histórico en el tercer grupo etario (55 y más años) de los integrantes de la muestra, lo que podría relacionarse con las modificaciones en el rendimiento de habilidades lingüísticas producto del envejecimiento, lo que, quizás, se vincula con los hallazgos de los estudios acerca de los efectos del envejecimiento en la estructuración sintáctica y discursiva que, en general, han dado cuenta de efectos en la memoria, el lenguaje y las funciones ejecutivas (cf. Véliz, 2004; Labos, Del Río y Zabala, 2009). En el caso del presente histórico, en particular, hay un cambio en la forma habitual de aludir al pasado. Este mismo hallazgo fue reportado por San Martín y Guerrero (2013) para algunas formas del discurso referido.

Asimismo, no deja de ser interesante que la disminución del empleo del presente histórico en los sujetos de 55 años y más de la muestra genera narraciones menos dinámicas que aquellas que sí lo incluyen, como veremos en los ejemplos de 4.2., porque este cambio de pretérito a presente histórico, en tanto mecanismo evaluativo, es el que, en parte, hace de la historia un relato mucho más entretenido. No obstante lo anterior, convendría proponer futuras investigaciones en las que se segmente el grupo de 55 años y más en subgrupos, ya que, en este caso, el rango de edades de los informantes de este grupo de edad es muy amplio (desde los 55 hasta los 87 años). Así, lo relevante sería comprobar en términos estadísticos que, efectivamente, si se avanza en la edad de este grupo de sujetos, disminuye el empleo de estos recursos de evaluación interna. De no comprobarse esta hipótesis, podría situarse este fenómeno solo en la dicotomía de las formas marcadas frente a las no marcadas, donde, por supuesto, el presente histórico sería la forma marcada. Finalmente, podría estudiarse la posibilidad de vincular este fenómeno con las formas de cortesía positiva de Brown \& Levinson (1987).

\subsubsection{Incidencia del factor grupo socioeconómico en el empleo del presente histórico}

En lo que respecta a la variable grupo socioeconómico, tampoco se encontró significatividad, $\mathrm{p}=0,417$. En este caso, el 27,3\% (96/351) de los usos del presente histórico se registra en los informantes del grupo bajo, 31,3\% (110/351) en los sujetos del grupo medio bajo, 28,6\% (100/351) en los del grupo medio y $12,8 \%$ (45/351) en los del grupo medio alto. Se presenta, a continuación, el Gráfico 3, de presencia y ausencia de presente histórico en la muestra analizada. 
Una aproximación sociolingüística al empleo del presente histórico en narraciones de experiencia personal en... / S. GUERRERO

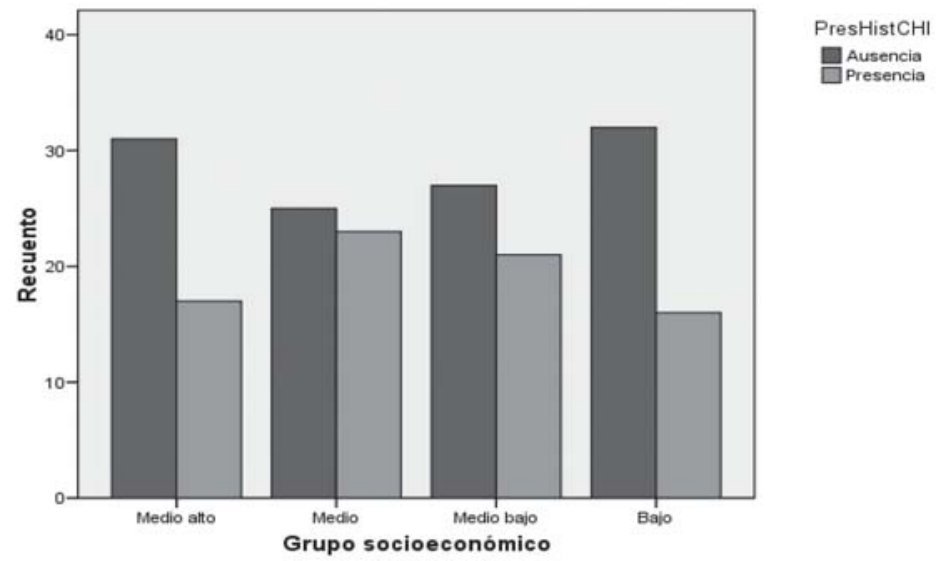

Gráfico 3. Presencia y ausencia de presente histórico en las narraciones de la muestra según el factor grupo socioeconómico.

Del gráfico precedente se destaca que, nuevamente, la ausencia del fenómeno en estudio es menor a su presencia en los cuatro grupos socioeconómicos. No obstante, es interesante que, al menos en la muestra analizada, las frecuencias más altas se concentran en los grupos medios de la escala social, esto es, en el grupo medio hay un $29,9 \%$ (23/77) de los informantes que lo emplea en tanto que los del grupo medio bajo lo utilizan en un 27,1\% (21/77) de los casos. En relación con el estrato alto, éste es usado por el 22\% (17/77) de los sujetos y en el estrato bajo por el 21\% (16/77) de los mismos. Sin embargo, al no haberse comprobado la significatividad, no se puede generalizar este resultado a la comunidad de habla en estudio. Consecuentemente, el posible patrón curvilíneo observado sólo es aplicable a la muestra en estudio.

\subsubsection{Análisis del empleo del presente histórico en correlación con las variables sociales en interacción}

Luego del análisis estadístico de variables cruzadas, se puede señalar que son las mujeres de 20 a 34 años y las de 55 años y más las que presentan significatividad estadística. Esto sugiere que las informantes femeninas de los dos grupos etarios mencionados y de los cuatro grupos socioeconómicos en estudio tienen este comportamiento no por azar, sino que podría tratarse, posiblemente, de un patrón en ellas. Ambos casos se ilustran, en términos de porcentajes, en los gráficos 4 y 5 , respectivamente. 
RLA. Revista de Lingüística Teórica y Aplicada, 52 (2), II Sem. 2014

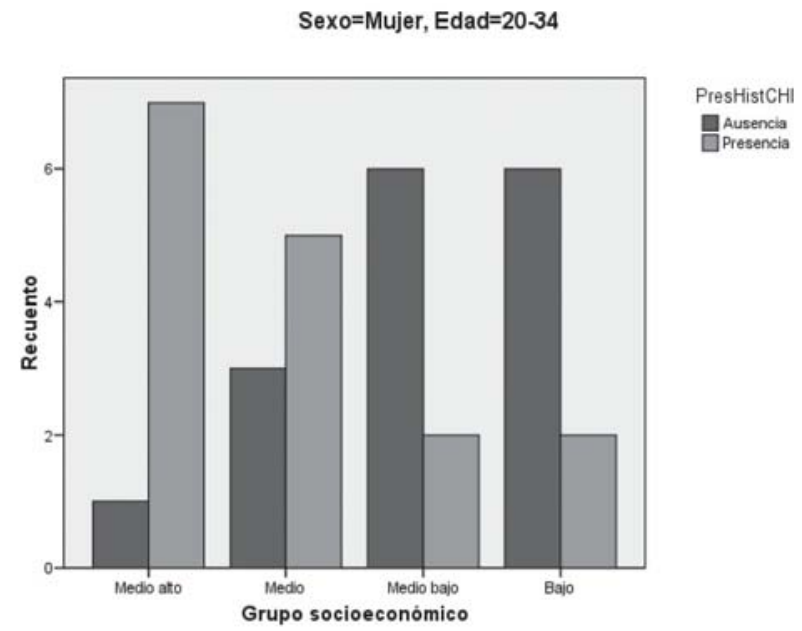

Gráfico 4. Gráfico ji cuadrado correspondiente a la interacción de la variables sexo, edad y grupo socioeconómico.

Como se desprende del Gráfico 4, el valor de ji cuadrado es menor a 0,05 $(\mathrm{p}=0,029)$. En este caso, la significatividad estadística de las variables independientes se genera al observar la distribución de los datos en los cuatro grupos socioeconómicos según el sexo y la edad. En consecuencia, en las mujeres de los grupos socioeconómicos más altos es mayor el uso del presente histórico.

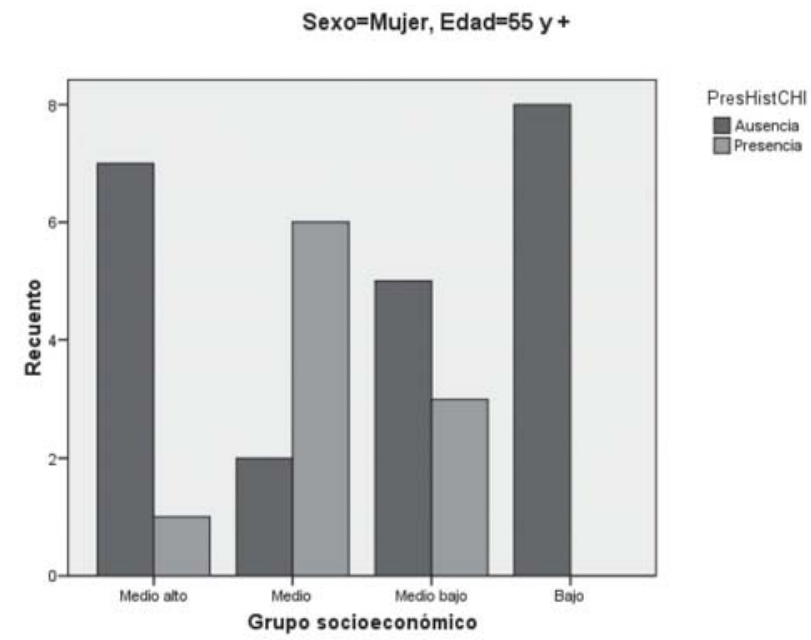

Gráfico 5. Gráfico ji cuadrado correspondiente a la interacción de la variables sexo, edad y grupo socioeconómico. 
De acuerdo con el gráfico que precede, lo mismo ocurre en el caso de las mujeres de 55 años y más, donde $\mathrm{p}=0,007$, muy por debajo del 0,05 , lo que significa que en los extremos de la escala socioeconómica el fenómeno en estudio se emplea muy poco (o no se emplea), a diferencia de los grupos intermedios, especialmente, en el medio, donde su presencia es muy marcada. Finalmente, cabe destacar que un valor muy cercano a la significatividad se encuentra en los hombres del primer grupo etario $(\mathrm{p}=0,114)$, aunque no alcanza la misma.

\subsection{Análisis del empleo y de la función de presente histórico según la estructura narrativa de Labov \& Waletzky (1967) y de Labov (1972)}

Por lo que se refiere al análisis del uso del presente histórico en la estructura narrativa, es preciso señalar que se consideraron los 351 casos donde se esperaba la aparición del pretérito. Cada uno de dichos casos fue observado en función de la sección de la estructura narrativa en la que se encontraba, siguiendo la propuesta de Labov \& Waletzky (1967) y de Labov (1972). Asimismo, en relación con los verba dicendi, que han sido estudiados desde el punto de vista del habla reportada y, por ende, como parte del componente evaluativo de las narraciones por dotarlas de mayor dinamismo (cf. Soler 2004; Jiménez, 2006; Guerrero, 2011a), hemos optado por incluirlo en el análisis atendiendo al comportamiento que adquieren en la narración. A fin de respaldar esta decisión, citamos a Avilés y Osorio (2010):

[los verbos dicendi] presentan un comportamiento especial en las narraciones, puesto que a veces se comportan como eventos, es decir, hacen avanzar el relato, mientras que otras veces actúan como pseudoeventos, esto es, suspenden la acción dramática. En los casos en que un verbo dicendi actúa como evento, la cláusula asociada al verbo 'decir' no se interpreta como el mero hecho de decir algo, sino como la realización de una acción -por ejemplo, 'le dijo ¿qué le pasó, compadre?' equivale a 'le preguntó $[\ldots]$ '-, mientras que cuando actúa como pseudoevento cumple netamente una función evaluativa, la que consiste básicamente en citas o comentarios atribuidos a los personajes de la narración del tipo 'entonces me dije [...]' (Avilés y Osorio, 2010: 73).

De acuerdo con el análisis, los 351 casos de presente histórico se ubicaron en la complicación de la narración ${ }^{5}$. Para realizar el conteo anterior, cada narración fue dividida en sus cláusulas constituyentes, a fin de estudiar la sección específica

${ }^{5}$ Estos datos respaldan los hallazgos de Silva-Corvalán (1983), quien señala que los eventos codificados en presente histórico aparecen en la sección de complicación, entendiendo por ésta tanto la acción complicada como la resolución de la propuesta laboviana, ya que ambas están constituidas por eventos narrativos. 
del relato con la que éstas se vinculan (Labov \& Waletzky, 1967). Así, las cláusulas encabezadas por formas verbales tanto en pretérito como en presente histórico fueron adscritas a determinadas secciones, según se observa a partir del análisis de la narración B I H002, en la que el entrevistado relata uno de los episodios más vergonzosos de su vida. En relación con el empleo del presente histórico, éste se presenta, en todos los casos, en el momento climático del relato y, por tanto, justifica el carácter reportable e inusual de la narración.

\section{Narración B I H002}

a. bueno la que más me hizo pasar vergüenza así como en público fue una vez que esta[b]a arri[b]a [d]el metro

b. mm [es] taba medio enfermo así agripa[d]o

c. esas enfermeda[d]eh normales que le dan a la persona

d. me sentía mal

e. me gano en frente de una abuelita que ehta[b]a senta[da] con un bastón

f. la abuelita no hay no halla na[d]a mejor que pararse a darme el asiento en el metro

g. mijito mire la cara anda que anda trayendo venga siéntese

h. así terrible $[\mathrm{d}] \mathrm{e}$ amable

i. cuando veo yo

j. me coloco rojo

k. me tapo la cara

1. y to[d]as las personas muertas [d]e la risa las que habían escucha[d]o arriba [d]el vagón

m. imagínate pa[ra] mí o sea rojo

n. la abuela me había da[d]o el asiento

o. yo abuelita gracias siéntese por favor

p. así no me haga pasar más vergüenza

q. que fue algo atroz

r. te lo juro

s. yo me bajé del metro porque no aguanté la risa de la gente así

Como se desprende de la narración, sólo las cláusulas $e, f, i, j, k$ y $s$ son relevantes para la cadena narrativa, vale decir, dan cuenta de eventos que ocurrieron en el mundo, puesto que mantienen entre ellas relaciones de tipo causal. De este modo, previo a la presentación del primer evento narrativo, el narrador entrega una serie de cláusulas de orientación $-a, b, c$ y $d$-tendientes a situar al oyente respecto a las coordenadas espacio-temporales en que se enmarca su vivencia para que ésta sea interpretada de manera correcta, esto es, como una de las más vergonzosas en las que se ha visto envuelto. Sólo una vez entregadas dichas indicaciones contextuales 
el hablante presenta el primer evento narrativo, a saber, el desplazamiento efectuado por él hacia una localización que resultará problemática en el transcurso de la narración -cláusula $e$. Una vez que se ha indicado este primer acontecimiento, el relato avanza dando cuenta de la reacción que este hecho provocó en un personaje clave de la historia -cláusula $f$-, junto con las posteriores reacciones experimentadas por el mismo protagonista-cláusulas $i, j, k$ y $s-$.

Resulta relevante que de las seis cláusulas narrativas antes mencionadas, cuatro de ellas estén encabezadas por formas verbales en presente histórico. Lo anterior obedece a que dicha forma verbal actúa como mecanismo de evaluación interna (Silva-Corvalán, 1983; Avilés y Osorio, 2010), puesto que se vincula con los eventos más reportables del relato y que, por tanto, justifican la narración de dicha experiencia. Como se observa en la narración anterior, aquella que, a juicio del hablante, más [le] hizo pasar vergüenza así como en público, los eventos destacados en presente histórico corresponden a los más críticos del relato y que contribuyen a la interpretación de la situación narrada como una de las más vergonzosas que ha experimentado. Esto permite explicar que el primer evento narrativo -cláusula $e$ sea destacado en presente histórico, ya que si bien un desplazamiento efectuado al interior del metro no resulta una acción inusual ni extraña en la vida del hablante, será aquella nueva ubicación adoptada la que generará las condiciones para que se produzca la situación que él califica como una de las más vergonzosas de su vida. En este sentido, el ganarse frente a la abuelita -cláusula $e$ - es lo que producirá que ésta le ceda el asiento.

Sin embargo, como se infiere de la narración presentada, no es la acción de la abuelita la que genera la vergüenza del protagonista -razón por la cual dicha cláusula aparece en pretérito y no en presente histórico-, sino que es la percepción que éste tiene de la reacción del público que observaba la situación: solo una vez que el protagonista $v e$, esto es, toma conocimiento de la risa que ha generado en los otros pasajeros es que experimenta vergüenza -cláusula $i-$, lo que explica que dicho evento sea indicado en presente histórico, puesto que corresponde a la situación que justifica y da sentido a la narración, cuyo tema era, precisamente, una de las anécdotas más vergonzosas que ha experimentado. Junto con la percepción de la situación en que se ha visto envuelto, el narrador marca en presente histórico las cláusulas $j \mathrm{y} k$, correspondientes a las acciones siguientes a tal percepción y que contribuyen a configurar la vivencia como una de las más vergonzosas que recuer$\mathrm{da}$, a saber, colocarse rojo y taparse la cara, respectivamente. Tal importancia tienen dichos eventos narrativos para la interpretación deseada por el hablante, que éste suspende la narración mediante una serie de comentarios evaluativos -cláusulas $m, n, o, p, q$ y $r-$, a través de los cuales enfatiza la vergüenza sentida ([...] o sea rojo [...]), calificándola explícitamente como algo atroz.

Una vez indicados en presente histórico los eventos cruciales para la interpre- 
tación deseada del relato, el hablante concluye su narración mediante la cláusula $s$, en la que señala el evento que pone fin a la experiencia-bajarse del metro- porque, según ha comentado a lo largo del relato, no aguant[ó] la risa de la gente así.

A partir del análisis anterior, que constituye sólo un ejemplo de las narraciones que presentan el fenómeno en estudio, hay que enfocarse en el hecho de que el presente histórico podría interpretarse como un fenómeno variable de la morfología, pero también con una dimensión discursiva. Como se vio, el fenómeno morfológico en estudio tiene incidencia en la construcción de discurso narrativo, específicamente como mecanismo de evaluación interna, cuya función es transformar la narración en reportable y que, por tanto, responden a una función evaluativa, dotando al discurso de mayor dinamismo y vivacidad. $\mathrm{Al}$ respecto, la propuesta de la Nueva gramática de la lengua española es un síntoma de este hecho, al proponer una distinción terminológica entre presente histórico y presente narrativo, aunque el fenómeno sigue siendo presente histórico en la definición más clásica y simple del fenómeno: uso del presente con valor de pasado. En consecuencia, el fenómeno aquí analizado es morfológico pero con valor de discurso, en particular en las evaluaciones de los relatos, cuyo efecto es narrar como si los hechos estuvieran ocurriendo ante los ojos de los interlocutores, tal como ocurre, por ejemplo, en el discurso referido. Esta idea, además, tiene sus antecedentes en los conceptos que Chafe (1982) denomina implicación (involvement) y distanciamiento (detachment) para referirse a los rasgos lingüísticos que diferencian el lenguaje oral del lenguaje escrito, respectivamente. Para Chafe, uno de los rasgos que daría cuenta de la implicación en el discurso oral es el presente histórico, lo que concuerda con la función retórica de la que veníamos hablando, esto es, la de vivacidad, pues en la medida en que se emplea este rasgo de involucramiento, la historia parece más real para sus interlocutores, tal y como si estuviera sucediendo en el presente.

En segunda instancia, aunque no fue el objetivo estudiar, mediante una comparación en sus ocurrencias, los dos valores que adquiere la forma de pasado (pretérito y presente histórico), se considera relevante este aspecto para una próxima investigación. En este sentido, la tensión entre ambas formas verbales, que tendrían igualdad funcional en la medida en que existe referencia a un hecho pasado en una narración, da cuenta de la posibilidad que tienen los informantes de "elegir" uno de dichos valores como medio de justificación del carácter reportable de la narración. Al emplear el presente histórico en el clímax de la historia, aun cuando se trata de un hecho pasado que, por tanto, pudo relatarse usando el pretérito, los sujetos le otorgan un valor especial al relato, a saber, justificarlo y, además, dotarlo de mayor dinamismo. Al respecto, si bien hubo sujetos que emplearon el presente histórico para este fin, hubo otros, en especial aquellos que se ubican en los extremos de la pirámide que emplearon muy poco o nada. Obsérvese, por ejemplo, las cláusulas de la complicación en el siguiente ejemplo: 
Una aproximación sociolingüística al empleo del presente histórico en narraciones de experiencia personal en... / S. GUERRERO

\section{Narración B III M042}

a. de mi hija mayor/ que al año tres meses le dio una pleuresía de un resfriado mal cuidado

b. le dio bronconeumonía

c. y el doctor no no conoció eso

d. y se le pasó a pleuresía

e. y [es] tuvo un año diez meses hospitaliza[d]a

f. ese fue el susto más grande que he teni[d]o

Como puede observarse, en el relato de B III M042, los eventos reportables -cláusulas $b, c y d$ - se encuentran encabezados por verbos en pretérito y no en presente histórico, como era de esperarse. De esta forma, la narración queda exenta del dinamismo que le otorga este rasgo, volviéndose, incluso, aburrida. En consecuencia, no llama la atención de quienes actuamos como receptores de la misma, porque los hechos no se narran como si estuvieran ocurriendo ante nuestros ojos.

\section{CONCLUSIONES}

Los hallazgos más relevantes de esta investigación se pueden sintetizar como sigue:

1. La posibilidad de considerar el presente histórico como un fenómeno variable. En esta investigación nos hemos hecho parte de la discusión que desde los sesenta se ha venido gestando en torno a la posibilidad de extender los estudios sobre variación sociolingüística más allá del nivel fonológico. En concreto, se considera el presente histórico como un fenómeno variable dentro del discurso narrativo, con una función específica, la de ser un recurso de evaluación interna dentro del relato. Consecuentemente, las investigaciones basadas en las narrativas para describir una comunidad de habla son de gran utilidad, con lo que queda demostrado que la perspectiva variacionista aplicada a la variación del discurso no puede limitarse exclusivamente a formas diferentes de decir lo mismo, sino que -sin duda- puede adoptar un enfoque variacionista más general.

2. Existe variación sociolingüistica solo en el cruce de las variables externas en correlación con el empleo del presente histórico en narraciones de experiencia personal en hablantes de Santiago de Chile. Si bien el análisis general del fenómeno muestra algunas tendencias en el empleo del presente histórico en el corpus, como la disminución de su frecuencia conforme avanza la edad o el mayor empleo en las mujeres, sólo se verificó la significación estadística en el análisis de las variables cruzadas, donde son las mujeres de 20 a 34 y las de 55 y más años las que mostraron significación estadística. En consecuencia, nuestra hipótesis sólo se 
verificó parcialmente.

3. El presente histórico es parte de la complicación en la narración. El análisis realizado demuestra que las cláusulas narrativas suelen estar encabezadas por formas verbales en presente histórico, obedeciendo a lo que Silva-Corvalán (1983) denomina evaluación interna. En este sentido, el presente histórico está directamente vinculado con los eventos más reportables del relato, y no solo le otorga vivacidad a la historia, sino que, además, justifica la narración de la misma.

4. Para finalizar, parece oportuno destacar el carácter limitado de los hallazgos y de las interpretaciones de la investigación aquí expuesta, en especial considerando que su enfoque es a nivel de variación discursiva, pero en el sentido genérico del término. Futuros trabajos respecto del fenómeno estudiado permitirán verificar la validez de estos hallazgos.

\section{REFERENCIAS}

Alarcos Llorach, Emilio. (1994). Gramática de la lengua española. Madrid: EspasaCalpe.

Avilés, Tania y Gabriela Osorio. (2010). Tiempo, aspecto y narración: Uso y función del presente histórico en narraciones de experiencia personal. Informe de tesis para optar al grado de Licenciada en Lengua y Literatura Hispánica con mención en Lingüística, Facultad de Filosofía y Humanidades, Universidad de Chile.

Bello, Andrés. (1841). Análisis ideológica de los tiempos de la conjugación castellana: reproducción facsimilar de la edición Príncipe, Valparaiso, 1841. Caracas, Venezuela: Plan Cultural Caracas.

Blas Arroyo, José Luis. (2005). Sociolingüistica del español. Desarrollos y perspectivas en el estudio de la lengua española en contexto social. Madrid, España: Cátedra.

Brown, Penélope \& Stephen Levinson. (1987). Politeness. Cambridge: Cambridge University Press.

Calsamiglia, Helena y Amparo Tusón. (1999). Las cosas del decir. Manual de análisis del discurso. Barcelona: Ariel.

Chafe, Wallace. (1982). Integration and involvement in speaking, writing, and oral literature. En D. Tannen (ed.), Spoken and written language. Exploring orality and literacy (pp. 35-53). Norwood, NJ, Estados Unidos: Ablex.

Coates, Jennifer. (2009). Mujeres, hombres y lenguaje. Un acercamiento sociolingüistico a las diferencias de género. México D.F, México: Fondo de Cultura Económica.

Gili Gaya, Samuel. (1961). Curso superior de sintaxis española. Barcelona, España: Bibliograf.

Guerrero, Silvana. (2011a). Diferencias de género en evaluaciones de narraciones 
Una aproximación sociolingüística al empleo del presente histórico en narraciones de experiencia personal en... / S. GUERRERO

de experiencias personales en el habla juvenil de Santiago de Chile. Una aproximación sociolingüística. Revista Signos. Estudios de Lingüistica, 44, 75, 18-32.

Guerrero, Silvana. (2011b). Análisis sociolingüístico de las diferencias de género en patrones narrativos de historia de experiencia personal en el habla juvenil de Santiago de Chile. Boletín de Filología, XLVI, 2, 85-106.

Guerrero, Silvana. (2013). Sobre la aplicación de la perspectiva sociolingüística al estudio de la variación discursiva: El caso de la narración de experiencia personal. Onomázein, 28, 188-200.

Jiménez, Teira. (2006). La narración infantil. Un estudio en niños de educación básica. Revista de Investigación, 60, 157-174.

Labos, Edith; Del Río, Miriam y Zabala, Karina. (2009). Perfil de desempeño lingüístico en el adulto mayor. Revista Argentina de Neuropsicología, 13, 1-13.

Labov, William. (1972). The transformation of experience in narrative syntax. En Language in the inner city. Studies in the black English vernacular, pp. 354-375. Filadelfia, Estados Unidos: University of Pennsylvania Press.

Labov, William. (1978). Where does the sociolinguistics variable stop? A response to Beatriz Lavandera. Texas Working Papers in Sociolinguistics, 44, 1-17.

Labov, William. (1983 [1972]). Modelos sociolingüisticos. Madrid, España: Cátedra.

Labov, William \& Waletzky, Joshua. (1967). Narrative analysis, en J. Helm (ed.), Essays on the verbal and visual arts (pp. 12-44). Seattle: University of Washington Press.

Lavandera, Beatriz. (1984 [1978]). Los límites de la variable sociolingüística. En Variación y significado (pp. 37-46). Buenos Aires, Argentina: Hachette.

Lenski, Gerhardt. (1954). Status crystalization: A non-vertical dimension of social status. American Sociological Review, 19, 405-413.

López Morales, Humberto. (2004). Sociolingüistica. Madrid: Gredos.

Moreno Fernández, Francisco. (1998). Principios de sociolingüística y sociología del lenguaje. Barcelona, España: Ariel.

Moreno Fernández, Francisco. (2009). Principios de sociolingüistica y sociología del lenguaje (cuarta edición). Barcelona: Ariel.

Moreno Fernández, Francisco. (2012). Sociolingüistica cognitiva. Proposiciones, escolios y debates. Madrid/Frankfurt, España/Alemania: Iberoamericana/Vervuert.

Poblete, María Teresa. (1998). Los marcadores discursivo-conversacionales de más alta frecuencia en el español de Valdivia (Chile). Estudios Filológicos, 33, 93103.

Prieto Vera, Luis. (1995-1996). Análisis sociolingüístico del dequeísmo en el habla de Santiago de Chile. Boletin de Filología, XXXV, 379-452.

Prieto Vera, Luis y San Martín, Abelardo. (2002-2003). Diferencias de género en el empleo del discurso referido: aproximación sociolingüística y pragmático- 
discursiva. Boletin de Filología, XXXIX, 269-303.

Real Academia Española. (2009). Nueva gramática de la lengua española (NGLE). Madrid/España: Espasa Calpe.

Reyes, Claudia. (1996). Estrategias narrativas en la zona metropolitana de Monterrey. En Rodríguez Flores y Rodríguez Alfano (eds.), Lenguaje y sociedad: Metodología y análisis aplicados a El habla de Monterrey (pp. 101-120). México: Trillas.

Reyes, Claudia. (2002). Algunas diferencias en las narraciones de hombres y mujeres dentro del corpus de "El habla de Monterrey". Iztapalapa, 53, 101-115.

Reyes, Claudia. (2003). Visión panorámica de los estudios sobre la narración. Revista de Humanidades: Tecnológico de Monterrey, 15, 95-119.

Rojas, Cristián; Rubio, Alejandra; San Martín, Abelardo, y Guerrero, Silvana. (2012). Análisis pragmático y sociolingüístico de los marcadores discursivos de reformulación en el habla de Santiago de Chile. Lenguas Modernas, 40, 103-123.

San Martín, Abelardo. (2004-2005). Igual como marcador discursivo en el habla de Santiago de Chile: función pragmático-discursiva y estratificación social de su empleo. Boletín de Filología, XL, 201-232.

San Martín, Abelardo. (2011). Los marcadores interrogativos de control de contacto en el corpus PRESEEA de Santiago de Chile. Boletín de Filología, XLVI (2), 135-166.

San Martín, Abelardo. (2013). Los reformuladores de distanciamiento en el corpus PRESEEA de Santiago de Chile. Boletín de Filología, XLVIII (1), 171-199.

San Martín, Abelardo y Silvana Guerrero. (2013). Una aproximación sociolingüística al empleo del discurso referido en el corpus PRESEEA de Santiago de Chile. Revista Signos, 46 (82), 258-282.

Serrano, María José. (2011). Sociolingüistica. Barcelona: Ediciones del Serbal.

Shiro, Martha. (2007). El discurso narrativo oral en la vida cotidiana: géneros y procesos. En A. Bolívar (ed.), Análisis del discurso (pp. 121-143). Caracas, Venezuela: Colección Minerva, Manuales Universitarios.

Silva-Corvalán, Carmen. (1983). Tense and aspect in oral spanish narrative: Context and meaning. Language, 59 (4), 760-780.

Silva-Corvalán, Carmen (2001). Sociolingüistica y pragmática del español. Washington, Estados Unidos: Georgetown University Press.

Soler, Sandra (2004). Discurso y género en historias de vida. Una investigación de relatos de hombres y mujeres en Bogotá. Bogotá: Publicaciones del Instituto Caro y Cuervo.

Valencia, Alba y Hermansen,Violy. (1985). El léxico de los estudiantes de cuarto año medio: El verbo. Materiales. Santiago, Chile: Universidad de Chile.

Véliz, Mónica. (2004). Procesamiento de estructuras sintácticas complejas en adultos mayores y adultos jóvenes. Estudios Filológicos, 39, 65-81. 